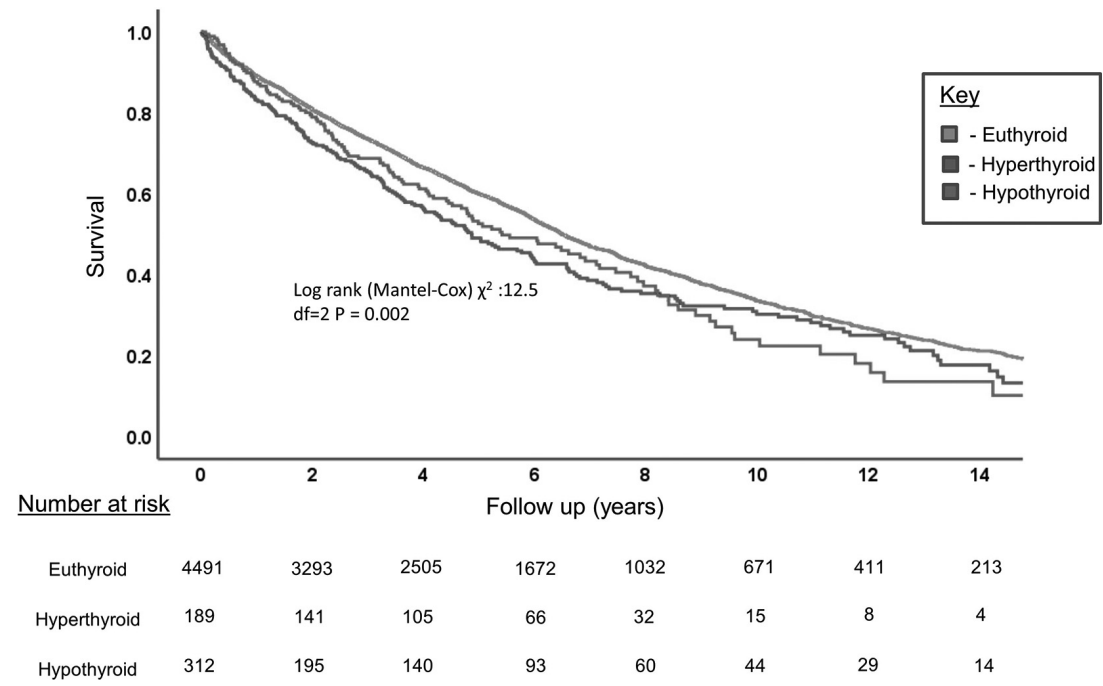

Abstract 91 Figure 2 Kaplan-Meier survival curve for all-cause mortality stratified by thyroid status

but the association disappeared in multivariable analysis (Table 2). The three strongest predictors of adverse outcome were age, increasing NT-proBNP, and higher NYHA class.

Conclusion Although thyroid dysfunction is associated with worse survival in patients with $\mathrm{CHF}$, it is not an independent predictor of mortality.

Conflict of Interest None

\section{THE IMPACT OF A NURSE-LED HEART FAILURE SERVICE ON COMPLEX DEVICE NEED}

John Graby*, Gemma Tibbs, Dawn Giblett, Mark Dayer, Oliver Gosling. Taunton and Somerset NHS Foundation Trust

\subsection{6/heartjnl-2019-BCS.90}

Introduction The prevalence of left ventricular systolic dysfunction (LVSD) continues to rise. It is recognised that optimal medical therapy can improve ejection fraction $(\mathrm{EF})$ in a proportion of patients. A nurse-led Heart Failure Service (HFS) was established in a district general hospital to deliver this, and previous analysis has demonstrated its efficacy in optimising HF drugs to recognised therapeutic doses.

Complex devices (Implantable Cardiac Defibrillators [ICDs] or Cardiac Re-synchronisation Therapy Devices [CRTs]) are established treatments for HF patients if on Optimal Medical Therapy (OMT). The European Society of Cardiology (ESC) and National Institute for Clinical Excellence (NICE) guidelines have expanded their role with a broad cohort of patients eligible for this therapy.

Aim We sought to determine how many patients, who at baseline had an indication for device therapy, improved after OMT to an extent that they no longer had an indication for device therapy.

Methods A retrospective analysis was undertaken of the HFS database over a 5 year period recording ECG for QRS duration \& morphology, surveillance of EF, device outcome.

Exclusion criteria: baseline EF $>35 \%$; device in-situ or listed for prior to HFS referral; no identifiable baseline EF or ECG.
Results 502 patients were analysed and 264 patients met criteria for a new device or upgrade at baseline - 191 male (72\%), 73 female (28\%), median age 73 (IQR $64-80$ ).

$76 / 97(78 \%)$ of potential CRT candidates (Pacing or Defibrillator component) were male. $16 / 97$ (16\%) improved to an EF $>35 \%$ (median age 68 [IQR 64-72], 14 male [88\%], 2 female [12\%]). 4/16 (25\%) of these patients underwent pharmacological or electrical cardioversion, which can contribute to EF improvement.

There were 167 potential ICD candidates (115 male [69\%]). 53/167 (32\%) improved to an EF >35\% (median age 68 [IQR 56-75], 33 male [61\%]). 7/53 (13\%) of these patients had other treatments that can also improve EF (cardioversion, 4; re-vascularisation, 2; valvuloplasty,1).

69/264 (26.1\%) patients did not require a device after optimal therapy had been delivered.

Conclusion We have demonstrated that in a significant proportion of HFS patients, the delivery of high quality OMT translates into an improvement in EF.

Not all patients that strictly 'meet guideline criteria' will be offered a device, nor will all patients take up offer of a device. However, this improvement in EF is likely to benefit the patient symptomatically and prognostically, and carry a significant cost saving to healthcare institutions. A cost analysis study will be undertaken to further assess this.

Conflict of Interest Nil

\section{IMPROVING AWARENESS AND ADHERENCE TO MEDICATIONS AMONGST HEART FAILURE PATIENTS IS THE MOST COST EFFECTIVE WAY OF REDUCING HEALTHCARE COSTS}

Syed Raza. Leighton Hospital, UK

\subsection{6/heartjnl-2019-BCS.91}

Background Heart Failure (HF) is a common medical condition and an important public health issue. This carries with it high mortality and frequent hospitalization. There is generally high re-admission rate and patients of heart failure tend to have a duration of stay in the hospital. Heart failure management is costly and puts a burden on healthcare budget. Lack 